\title{
Galaxy scaling relations from the ACS Virgo and Fornax Cluster Surveys: no evidence for a dwarf-giant dichotomy
}

\section{Patrick Côté $^{1}$, Laura Ferrarese ${ }^{1}$, Andrés Jordán ${ }^{2}$, John P. Blakeslee ${ }^{3}$, Chin-Wei Chen ${ }^{1,4}$, Leopoldo Infante ${ }^{5}$, Simona Mei ${ }^{6}$, Eric W. Peng ${ }^{1}$, John L. Tonry ${ }^{7}$ and Michael J. West ${ }^{2}$}

${ }^{1}$ Herzberg Institute of Astrophysics, Victoria, BC, Canada; ${ }^{2}$ European Southern Observatory, Garching, Germany; ${ }^{3}$ Washington State University, Pullman, WA, U.S.A.; ${ }^{4}$ National Central University, Jhongli, Taiwan; ${ }^{5}$ Pontificia Universidad Católica de Chile, Santiago, Chile; ${ }^{6}$ Observatoire de Paris, Paris, France; ${ }^{7}$ Institute for Astronomy, Honolulu, HI, U.S.A.

\begin{abstract}
We examine the photometric and structural properties of early-type galaxies derived from the ACS Virgo and Fornax Cluster Surveys, supplementing these data with previously published or newly reanalysed data for additional early-type galaxies in Virgo, Fornax and the Local Group. As we have noted elsewhere, Sérsic models are found to provide accurate representations of the observed brightness profiles on scales greater than a few percent of the effective radius, $R_{e}$. On smaller scales, the brightness profiles of bright $\left(M_{B} \gtrsim-20\right)$ galaxies show central deficits with respect to the inward extrapolation of the Sersic models; fainter galaxies usually show central excesses. Fainter than $M_{B} \approx-20$, we find a continuity in the photometric and structural scaling relations: i.e., between absolute magnitude, central surface brightness, Sérsic index, effective radius, and the surface brightness measured at, and averaged within, the effective radius. There is no evidence for a "dwarf/giant dichotomy" - a conclusion consistent with a number of recent studies but contrary to some earlier claims.
\end{abstract}

Keywords. galaxies: elliptical and lenticular, $\mathrm{cD}$ - galaxies: fundamental parameters - galaxies: nuclei - galaxies: photometry - galaxies: structure

\section{Introduction}

It is long been believed that early-type galaxies can be neatly separated into distinct "giant" and "dwarf" classes, with a division at $M_{B} \sim-18$. The widely accepted view that early-type/elliptical galaxies obey completely different photometric and structural scaling relations was introduced by Kormendy (1985) who argued that "there is a large discontinuity in the parameter correlations for bright [giant] ellipticals, including M32, and dwarf spheroidals [dwarfs]" and concluded that these two classes of galaxies "were formed in very different ways".

However, as noted by Graham \& Guzmán (2003a), the Kormendy (1985) sample had a marked absence of galaxies with $M_{B} \sim-18$ : the canonical dividing point between the alleged dwarf and giant populations (Jerjen \& Binggeli 1997 expressed similar concerns). In recent years, as high-quality photometric data have become available for more and more early-type galaxies, the reality of this dichotomy has been questioned in a number of studies (see also early contributions from Binggeli, Sandage \& Terenghi 1984 and Caldwell 1983, 1987): e.g., Jergen \& Binggeli (1997), Jerjen, Binggeli \& Freeman (2000), Graham \& Guzmán (2003), Graham et al. (2003ab), Gavazzi et al. (2005), Aguerri et al. (2005), Zibetti et al. (2005), Côté et al. (2006), Ferrarese et al. (2006a). In this article, we present new evidence from the ACS Virgo and Fornax Cluster Surveys - supplementing 
these data with structural parameters for additional faint galaxies in Virgo, Fornax and the Local Group — that there is a continuity in the photometric and structural properties of early-type galaxies for $M_{B} \gtrsim-20$.

\section{The ACS Virgo and Fornax Cluster Surveys}

The ACS Virgo and Fornax Cluster Surveys (Côté et al. 2004; Jordán et al. 2007) consist of HST imaging for 143 members of the Virgo and Fornax Clusters, all previously classified on the basis of ground-based photographic imaging to have early-type morphologies (e.g., types E, S0, dE, dE,N or dS0; Binggeli, Sandage \& Tammann 1985; Ferguson 1989). All HST images were taken with the ACS (Ford et al. 1998) using a filter combination equivalent to the $g$ and $z$ bandpasses in the SDSS photometric system. The images cover a $200^{\prime \prime} \times 200^{\prime \prime}$ field with $\approx 0.1^{\prime \prime}$ resolution, corresponding to a physical resolution of $\approx 8-9$ pc at the distances of Virgo and Fornax. The Virgo sample is complete for early-type galaxies brighter than $M_{B} \approx-19.2$ and $44 \%$ complete down to its limiting magnitude of $M_{B} \approx-15.2$, while the Fornax sample is complete down to $M_{B} \approx-16.1$.

For each galaxy, azimuthally averaged surface brightness profiles were derived as described in Ferrarese et al. (2006a). As we have noted elsewhere (e.g., Ferrarese et al. 2006abc; Côté et al. 2006, 2007; Jordán et al. 2007), there is systematic behavior of the brightness profiles on small scales (see Figure 1a): galaxies brighter than $M_{B} \sim-20$ typically show central light deficits with respect to the inward extrapolation of the best-fit Sérsic (1968) model, while the great majority of low- and intermediate-luminosity galaxies $\left(-19.5 \lesssim M_{B} \lesssim-15\right)$ show central light excesses. Galaxies occupying a narrow range of intermediate luminosities $\left(-20 \lesssim M_{B} \lesssim-19.5\right)$ are reasonably well fitted by Sérsic models over all radii (see also Graham et al. 2003b).

\section{A Continuity of Structural Properties: No Dwarf-Giant Dichotomy}

Panel (b) of Figure 1 shows the relationship between absolute blue magnitude, $M_{B}$, and central surface brightness, $\mu_{B, 0}$, for the Virgo and Fornax galaxies. $\dagger$ Absolute magnitudes have been derived using the SBF distances from Mei et al. (2007) and Blakeslee et al. (2008, in preparation). For bright galaxies $\left(M_{B} \lesssim-20\right)$, core-Sérsic models have been used to fit the profiles; for galaxies fainter than $M_{B} \sim-20$ (which often contain a nuclear excess), a double Sérsic parameterization was used to fit the entire profiles. Thus, central surface brightnesses have been measured using the inward extrapolation of the outer Sérsic component in nucleated galaxies (i.e., excluding the central excess) or using a single Sérsic model for galaxies that are well fitted by such models over all radii. Panel (c) of Figure 1 shows the corresponding relationship between $M_{B}$ and Sérsic index, $\mathrm{n}_{s}$.

Also included in panels $b c$ of Figure 1 are 23 early-type dwarf galaxies in Virgo and Fornax from Stiavelli et al. (2001). For consistency, we have re-fitted the WFPC2 profiles for these galaxies using the same family of models used for the ACS samples: i.e., single Sérsic models for non-nucleated galaxies and double Sérsic models for galaxies with nuclear excesses. At still lower luminosities, we plot the results of fitting Sérsic models to the brightness profiles of six M31 dSph galaxies from McConnachie \& Irwin (2006). In these cases, the surface brightness at $0.05^{\prime \prime}$ is measured after shifting the profiles to the distance of Virgo. Finally, we include 201 of the 226 early-type (i.e., dE or E) Virgo

$\dagger$ Measured at $R=0.05^{\prime \prime}$, equivalent to one ACS/WFC pixel, or $\approx 4-5$ pc at the distance of Virgo/Fornax. 
(a)

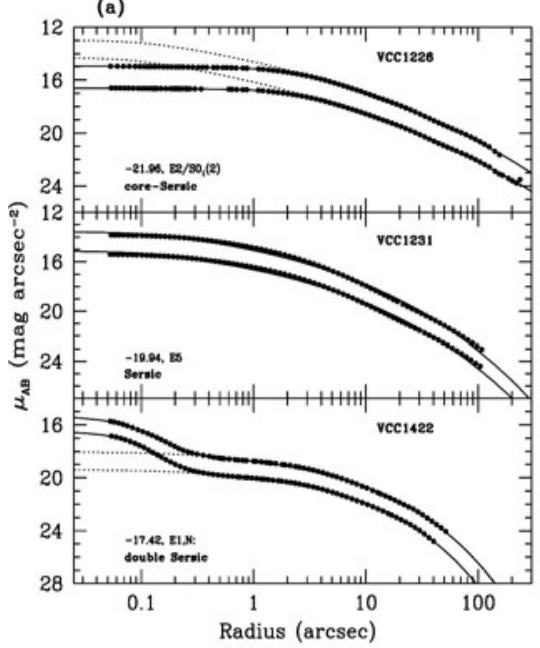

(b)

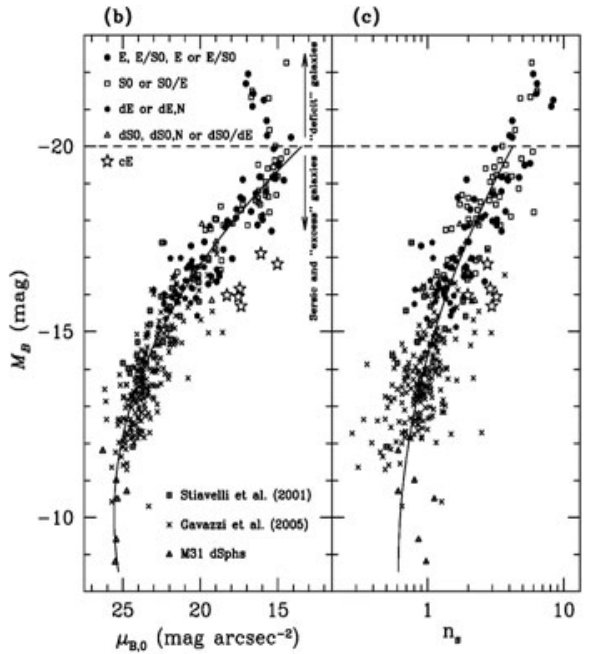

Figure 1. (Panel a) Surface brightness profiles in $g$ and $z$ for three galaxies from the ACS Virgo Cluster Survey, illustrating the transition from central "deficit" to "excess" relative to the inward extrapolation of Sérsic models that fit the outer profiles (dotted curves). (Panel b) Relationship between absolute blue magnitude, $M_{B}$, and the intrinsic $B$-band central surface brightness for early-type galaxies from the ACS Virgo and Fornax Cluster surveys. Also shown are $\mathrm{dE}$ and E Virgo galaxies from Gavazzi et al. (2005), early-type dwarfs from Stiavelli et al. (2001) and M31 dSphs from McConnachie \& Irwin (2006). The smooth curve shows the best-fit quadratic relation for galaxies fainter than $M_{B}=-20$ (indicated by the dashed line). (Panel $c$ ) Absolute blue magnitude plotted against Sérsic index, $\mathrm{n}_{S}$. The best-fit quadratic relation is shown by the smooth curve. The symbols are the same as in the previous panel.

galaxies observed by Gavazzi et al. (2005) with the 2.5m Isaac Newton Telescope, (i.e., omitting 25 galaxies that were already included in the ACS or WFPC2 samples).

The smooth curves in Figures $1 b$ and $1 c$ show the best-fit quadratic relations for the combined sample. Since the "deficit" galaxies are thought to have central cores depleted by supermassive black hole interactions, we have excluded these galaxies from the analysis. We have also excluded six Virgo or Fornax galaxies that have, at various times, been classifed as members of the rare class of "compact ellipticals" (cEs): VCC1297, VCC1199, VCC1192, VCC1440, VCC1627 and FCC143. Such galaxies are thought to have had their internal structure heavily modified by tidal interactions with nearby companions; indeed, all of these galaxies, except for VCC1440, are observed to have a bright, nearby companion with a similar velocity and SBF distance. These objects aside, we see from Figure 1 that there is a clear, continuous sequence in both panels extending from $M_{B} \sim-20$ down to the level of the M31 dwarfs - a range of more than 11 mag.

Figure 2 shows three additional scaling relations for this same sample: the dependence on absolute blue magnitude of (1) mean surface brightness within the effective radius, $\langle\mu\rangle_{e} ;(2)$ surface brightness measured at the effective radius, $\mu_{e}$; and (3) the effective radius, $R_{e}$. Once again, fainter than $M_{B} \sim-20$, there is no discontinuity in any of these relations. Moreover, as noted by Graham \& Guzman (2003a) the expected parameter correlations for Sérsic models can be calculated directly from the $M_{B}-\mu_{0}$ and $M_{B}-\mathrm{n}_{s}$ relations shown in Figures $1 b c$. We show these relations as the smooth curves in each panel. We conclude that there is no evidence for a "dwarf/giant dichotomy". 


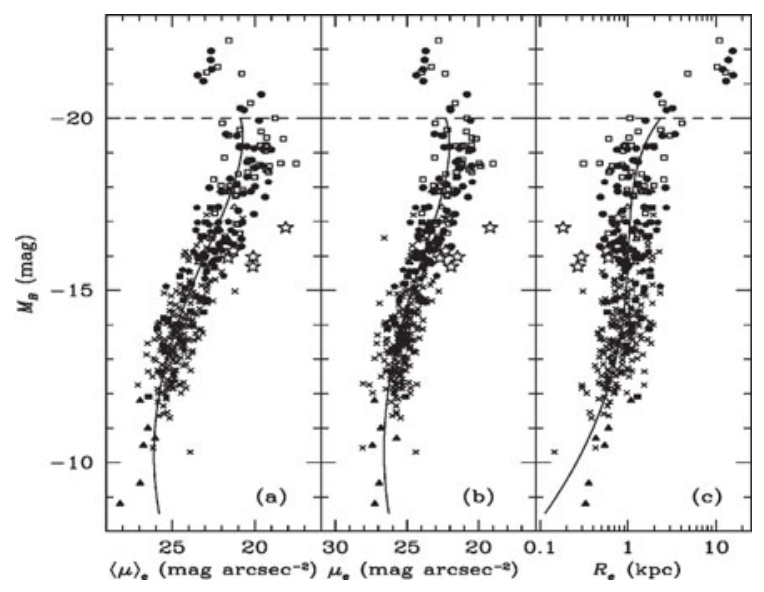

Figure 2. Dependence on $M_{B}$ of the mean $B$-band surface brightness inside the effective radius (panel $a), B$-band surface brightness measured at the effective radius (panel $b$ ), and effective radius (panel c). The symbols are the same as in the Figure 1 . The solid curves show the expected relations for Sérsic models, beginning with the $M_{B}-\mu_{0}$ and $M_{B}-\mathrm{n}_{s}$ relations in Figures $1 b c$.

\section{Acknowledgements}

We thank Massimo Stiavelli and Alan McConnachie for kindly providing the brightness profiles for the low-luminosity galaxies in Virgo, Fornax and M31.

\section{References}

Aguerri, et al. 2005, AJ, 130, 475

Binggeli, B., Sandage, A., \& Tammann, G. A. 1985, AJ, 90, 1681

Binggeli, B., Sandage, A., \& Terenghi, M. 1984, AJ, 89, 64

Caldwell, N. 1983, AJ, 88, 804

Caldwell, N. 1987, AJ, 94, 1116

Côté, P., et al. 2004, ApJS, 153, 223 (ACSVCS Paper I)

Côté, P., et al. 2006, ApJS, 165, 57 (ACSVCS Paper VIII)

Côté, P., et al. 2007, ApJ, in press (ACSFCS Paper II)

Ferguson, H. C. 1989, AJ, 98, 367

Ferrarese, L., et al. 2006a, ApJ, 164, 334 (ACSVCS Paper VI)

Ferrarese, L., et al. 2006b, ApJ, 644, L21

Ferrarese, L., et al. 2006c, arXiv:astro-ph/0612139

Ford, H. C., et al. 1998, Proc. SPIE, 3356, 234

Graham, A. W., et al. 2003a, AJ, 125, 2936

Graham, A. W., et al. 2003b, AJ, 125, 2951

Graham, A. W. \& Guzmán, R. 2003, AJ, 125, 2936

Gavazzi, G., et al. 2005, A\&A, 430, 411

Jerjen, H. \& Binggeli, B. 1997, in ASP Conference Series, Vol. 116, p. 239

Jordán, A., et al. 2007, ApJS, 169, 213 (ACSFCS Paper I)

Kormendy, J. 1985, ApJ, 295, 73

McConnachie, A. W. \& Irwin, M. J. 2006, MNRAS, 365, 1263

Mei, S., et al. 2007, ApJ, 655, 144 (ACSVCS Paper XIII)

Sérsic, J.-L. 1968, Atlas de Galaxias Australes (Córdoba: Obs. Astron., Univ. Nac. Córdova)

Stiavelli, M., et al. 2001, AJ, 121, 1385

Zibetti, S., et al. 2005, in IAU Colloq. 198, p. 380 